\title{
Editorial
}

\section{Atracurium and succinylcholine on the masseter muscle}

Three papers in this issue ${ }^{1-3}$ make an admirable attempt to correlate a common neuromuscular monitoring practice, observation of the force of contraction of the adductor pollicis, with muscle function at a site of particular clinical interest, the masseter. The masseter is the most powerful muscle influencing jaw closure and perhaps the most easily monitored muscle innervated by the fifth cranial nerve. Knowledge of its sensitivity to neuromuscular blocking drugs would have important implications for airway management during onset and recovery from paralysis, and for intraoperative monitoring of cranial nerve function. Careful documentation of the response of the masseter to succinylcholine might contribute to a better understanding of masseter spasm and its anaesthetic implications.

Function of the masseter can be evoked by electrical stimulation in front of the ear just below the zygoma. Function of the orbicularis oculi can be evoked by stimulation at a slightly more cephalad site, just above the zygoma. Previous studies of the orbicularis oculi suggest that this muscle is relatively resistant to the effects of atracurium compared with the adductor pollicis. ${ }^{4}$ In contrast, Saddler et al. ${ }^{1}$ documented that the masseter was as sensitive as the adductor pollicis to the effects of atracurium. Therefore one might hope to estimate reasonably well the degree of neuromuscular blockade in the masseter by observation of the function of the adductor pollicis.

However, these authors point out that the relative potency of atracurium at these two sites varied. The $\mathrm{ED}_{95}$ at the masseter was as little as 64 per cent and as much as 141 per cent of the $\mathrm{ED}_{95}$ at the adductor pollicis. In six of the ten patients the difference in potency between the two sites was greater than 20 per cent. These data differ from the results of a very similar study in adults, ${ }^{5}$ which found

Associate Professor of Anesthesiology, Department of Anesthesiology, Children's Hospital of Pittsburgh, and the University of Pittsburgh School of Medicine, Pittsburgh, PA 15213-2583 only two of ten patients in whom the thumb was more paralyzed than the jaw muscles following administration of pancuronium. The data suggest that the method of monitoring the function of the masseter used in these studies is more difficult to apply in children than in adults, and that measures of neuromuscular function of the masseter are more variable than measures of adductor pollicis function in children.

Nevertheless, onset of neuromuscular paralysis was uniformly more rapid in the masseter than in the adductor pollicis of these children. ${ }^{1-3}$ This is further evidence to suggest that the response of the adductor pollicis can be used clinically to predict the response of the masseter following a bolus of nondepolarizing neuromuscular blocking drug. This conclusion is consistent with many observations of intubation conditions during halothane anaesthesia and atracurium-induced paralysis which found adequate visualization of the larynx when the neuromuscular function of the adductor pollicis was depressed by at least $80-90$ per cent.

Given that the response of the masseter to the effects of a nondepolarizing neuromuscular blocking drug varied little from the response of the standard monitoring site, the adductor pollicis, the report of Plumley et al. ${ }^{2}$ is particularly interesting. They found that the doseresponse relationship of succinylcholine differed by 30 per cent at these two sites, with the masseter more sensitive than the adductor pollicis. There are several differences in methods between the dose-response studies of atracurium and of succinylcholine which should have increased the likelihood that the investigators would observe a difference in the response to atracurium, and decreased the likelihood of observing a difference in response to succinylcholine. Therefore, there can be no question about the conclusion: if the adductor pollicis is paralyzed after administration of an intubating dose of succinylcholine ( 1 to $2 \mathrm{mg} \cdot \mathrm{kg}^{-1}$, or 2.3 to 4.5 times the $\mathrm{ED}_{95}$ at the adductor pollicis) so is the masseter. This is an important documentation and qualification. Individuals with elevated pseudocholinesterase activity ${ }^{6}$ might be resistant to the effects of succinylcholine. In any case, 
lack of neuromuscular function is not necessarily equivalent to flaccidity after administration of succinylcholine.

The most interesting finding of these studies ${ }^{2,3}$ was that resting tension in the masseter increased in a dose-related manner as succinylcholine blocked neuromuscular function. A similar but less pronounced increase in resting tension was observed in the adductor pollicis. These observations have been foreshadowed by other studies. ${ }^{7}$ Using a different method, that did not include neural stimulation, Van Der Spek et al. observed a significant increase in stiffness of the muscles influencing mouth opening after administration of succinylcholine.$^{8-10}$

Increased resting tension or stiffness of the muscles moving the mandible can make laryngoscopy difficult. Plumley et $a l^{2}$ reported that the time (average plus or minus one standard deviation) from injection of succinylcholine to maximum increase in resting tension of the masseter was 15 to 70 seconds, while the duration of the effect was 10 to 120 seconds. In many patients, therefore, the effect of succinylcholine on resting tension, as measured in this study, could diminish before laryngoscopy is attempted. In other patients, the effect could be maximal during attempted laryngoscopy. The magnitude of this effect was directly dose-related. After administration of $1 \mathrm{mg} \cdot \mathrm{kg}^{-1}$ of succinylcholine the increase in resting tension in the jaw muscles was up to $90 \mathrm{~g}$ (one standard deviation above the mean). If the jaw opened as a perfect spring, this increase would appear to be too small to impede laryngoscopy. However, "considerable individual variability" in the effect of succinylcholine on resting tension of the masseter was noted. ${ }^{2}$ Van Der Spek et al. ${ }^{8}$ reported a 44 -fold range of increases in jaw stiffness after administration of succinylcholine. In several patients this increase in resistance to mouth opening was great enough to impede laryngoscopy ${ }^{8,9}$ The methods used by these investigators ${ }^{2.3}$ did not assess ease of mouth opening. Therefore, it is not known if the greatest increase in resting tension they noted could have been detected by the anaesthetist opening the mouth.

Those anaesthetists who have been unable to open the mouth of a patient after administration of succinylcholine might claim that these studies ${ }^{2,3}$ are measuring background noise rather than something related to masseter spasm. Perhaps masseter spasm, defined as inability to open the jaw caused by increased tension in the masseter after administration of succinylcholine, is an extreme case of the apparently normal dose-related increase in resting tension observed in these studies. More complete evaluation of the force required to open the mouth is necessary to clarify this issue.

If the occurrence of masseter spasm were due to an excessive increase in resting tension, then it would be reasonable to expect that patients at increased risk of masseter spasm might exhibit a greater increase in resting tension after administration of succinylcholine than other patients. Saddler et $a l^{3}{ }^{3}$ studied eight children with strabismus, a condition said to be associated with increased risk of masseter spasm, and found no difference in their increase in resting tension after succinylcholine compared with that in other children. It would appear that either these children are not more susceptible to masseter spasm, or the factors predisposing to masseter spasm are not reflected by these measurements of resting tension. In an earlier retrospective study claiming an increased incidence of masseter spasm in children with strabismus, ${ }^{11}$ the number of cases identified was small and the incidence of masseter spasm only 2.8 per cent or 1 in 35 . If the power of these measures of change in resting tension ${ }^{2,3}$ is similar to that of the retrospective identification of masseter spasm, ${ }^{11}$ then to identify an event related to masseter spasm in a population of eight, its incidence would have to be greater than 20 per cent. These investigators were not lucky enough to observe a rare event in the small population of eight children.

Perhaps masseter spasm is not only quantitatively different from the changes in resting tension measured here, ${ }^{8,9}$ but also qualitatively different. If so, the identifying features of masseter spasm have yet to be described in sufficient detail to allow differentiation of masseter spasm from an extreme instance of the normal increase in resting tension of the masseter following administration of succinylcholine.

In summary, these three studies ${ }^{1-3}$ quantify aspects of the neuromuscular function of the masseter that are relevant to the effective use of neuromuscular blocking drugs in the practice of anaesthesia. The dilemma of relatively high incidence of masseter spasm, ${ }^{11,12}$ the 50 per cent incidence of muscle biopsy results suggesting susceptibility to malignant hyperthermia in patients with a history of masseter spasm, ${ }^{13-15}$ and the low incidence of malignant hyperthermia ${ }^{16}$ cannot be resolved by these studies alone. Nevertheless, these three papers have made an important contribution to our understanding of the effects of neuromuscular blocking drugs in anaesthetized children by identifying a dose-related increase in resting tension of the masseter following administration of succinylcholine in normal children anaesthetized with halothane, and documenting the relative sensitivities of the adductor pollicis and the masseter to both succinylcholine and a nondepolarizing neuromuscular blocker. 


\section{Atracurium, et succinylcholine et le muscle masséter}

Dans le présent numéro, trois études ${ }^{1-3}$ tentent admirablement de corréler la force de contraction de l'adducteur du pouce, fréquemment employée pour surveiller la fonction neuromusculaire, avec la fonction musculaire à un site cliniquement intéressant, le masséter. Le masséter est le muscle le plus puissant de la mâchoire et peut-être le plus facile à surveiller des muscles innervés par le cinquième nerf crânien. Connaître sa sensibilité aux bloqueurs neuromusculaires aura des implications importantes sur le management des voies aériennes pendant l'installation et la récupération de la paralysie ainsi que pour la surveillance peroperatoire de la fonction des nerfs crâniens. Une documentation soignée de la réponse du masséter à la succinylcholine peut contribuer à une meilleure compréhension du spasme du masséter et ses implications anesthésiques.

La fonction du masséter peut être évoquée par une stimulation électrique en avant de l'oreille juste en bas de l'os malaire. La fonction de l'orbiculaire peut être évoquée par une stimulation légèrement plus céphalade à ce site juste en haut de l'os malaire. Des études antérieures sur l'orbiculaire suggèrent que ce muscle est relativement résistant aux effets de l'atracurium comparativement à l'adducteur du pouce. ${ }^{4}$ Par contre, Saddler et al. ' ont prouvé que le masséter était aussi sensible que l'adducteur du pouce à l'atracurium. Ainsi on peut espérer estimer raisonnablement le degré du bloc neuromusculaire du masséter par l'observation de la fonction de l'adducteur du pouce.

Cependant ces auteurs rappellent que la puissance relative de l'atracurium à ces deux sites varie. $\mathrm{La}^{\mathrm{E}} \mathrm{D}_{95}$ du masséter était aussi peu que 64 pour cent et aussi grande que 141 pour cent de celle de l'adducteur du pouce. Chez six sur dix patients la différence de puissance entre les deux sites était plus grande que 20 pour cent. Ces données diffèrent des résultats d'une étude similaire faite chez des adultes $^{5}$ qui trouve que seulement deux sur dix patients chez qui le pouce était plus paralysé que la mâchoire après administraiton de pancuronium. Les données suggèrent que la méthode de surveillance de la fonction du masséter était plus difficilement applicable chez les enfants comparativement aux adultes et que la mesure de la fonction neuromusculaire du masséter est plus variable que la mesure de la fonction de l'adducteur du pouce chez les enfants.

Cependant, l'installation de la paralysie neuromusculaire était plus rapide au niveau du masséter que l'adducteur du pouce chez les enfants. ${ }^{I-3}$ Ceci représente une évidence de plus que la réponse de l'adducteur du pouce peut être utilisée cliniquement pour prédire la réponse du masséter après un bolus de bloqueur neuromusculaire non-dépolarisant. Cette conclusion est en accord avec plusieurs observations des conditions d'intubation lors de l'anesthésie à l'halothane et une paralysie induite par l'atracurium. Une visualisation adéquate du larynx est alors observée quand la fonction neuromusculaire de l'adducteur du pouce était diminuée à 80-90 pour cent.

Etant donné que la réponse du masséter au bloc neuromusculaire non-dépolarisant varie peu de celle de l'adducteur du pouce, l'étude de Plumley et al. ${ }^{2}$ est particulièrement intéressante. Ils ont trouvé que la relation dose-réponse de la succinylcholine diffère de 30 pour cent aux deux sites, le masséter étant plus sensible que l'adducteur du pouce. Plusieurs différences méthodologiques entre les études de dose-réponse de l'atracurium et de la succinylcholine peuvent augmenter la probabilité pour que les investigateurs trouvent une différence dans la réponse à l'atracurium et diminuent les probabilités d'observer une différence celle à la succinylcholine. Ainsi, il n'y aucun doute sur la conclusion : si l'adducteur du pouce est paralysé après des doses d'intubation de succinylcholine (1-2 mg $\mathrm{kg}^{-1}$, ou 2.3 à 4.5 fois la $E D_{95}$ de l'adducteur du pouce) il en sera de même pour le masséter. Ceci représente une preuve importante. Les personnes ayant une activité accrue de la pseudocholinestérase ${ }^{6}$ peuvent être résistantes aux effets de la succinylcholine. Dans tous les cas l'abolition de la fonction neuromusculaire n'est pas nécessairement équivalente à la flaccidité après succinylcholine.

La trouvaille la plus intéressante de ces études ${ }^{2,3}$ est que la tension de repos du masséter augmente d'une façon dépendante de la dose quand la succinylcholine est utilisée. Une augmentation similaire mais moins prononcée était observée pour l'adducteur du pouce. Ces observations ont été mises à l'ombre par d'autres études. ${ }^{7}$ Utilisant une méthode différente qui n'induit pas une stimulation nerveuse, Van Der Spek et al. ont observé une augmentation significative des muscles influencant l'ouverture de la bouche après administration de succinylcholine..$^{8-10}$

L'augmentation de la tension de repos ou de la rigidité des muscles de la mâchoire peut rendre la laryngoscopie difficile. Plumley et $a .^{2}$ ont rapporté que le temps (moyenne \pm 1 ) à partir de l'injection de la succinylcholine au maximum d'augmentation de la tension de repos du 
masséter était de 15-70 secondes, alors que la durée des effets était de 10-120 secondes. Ainsi chez plusieurs patients, les effets de la succinylcholine sur la tension de repos, telle que mesurée dans cette étude, peut diminuer avant que la laryngoscopie soit tentée. Chez d'autres patients, l'effet peut être maximal durant la laryngoscopie. La magnitude de cet effet était directement reliée à la dose. Après administration de $1 \mu \mathrm{g} \cdot \mathrm{kg}^{-1}$ de succinylcholine l'augmentation de la tension de repos des muscles de la mâchoire était de $90 \mathrm{gr}$ (1 SD de plus que la moyenne). Si la mâchoire s'ouvrait en ressort, cette augmentation apparaîtrait non-significative pour empêcher la laryngoscopie. Cependant, la variabilité individuelle considérable dans l'effet de la succinylcholine sur la tension de repos du masséter était notée. ${ }^{2}$ Van Der Spek et al. ${ }^{8}$ ont rapporté une augmentation de 44 pour cent dans la rigidité de la mâchoire après administration de succinylcholine. Chez plusieurs patients cette augmentation de la résistance à l'ouverture de la bouche était assez grande pour empêcher la laryngoscopie. ${ }^{8,9}$ Les méthodes utilisées par les investigateurs ${ }^{2,3}$ n'ont pas évalué la facilité de l'ouverture de la bouche. Ainsi, on ne saura pas si le maximum d'augmentation de la tension de repos était détecté par l'anesthésiste lors de l'ouverture de la bouche. Les anesthésistes qui étaient incapables d'ouvrir la bouche des patients après succinylcholine peuvent dire que ces études ${ }^{2,3}$ mesurent le «bruit de fond " plutôt que quelque chose reliée au spasme du masséter. Peut-être que le spasme du masséter tel que défini par l'incapacité d'ouvrir la bouche à cause d'une augmentation de la tension dans le masséter après administration de succinylcholine est un cas extrême de l'augmentation normale de la tension observée dans ces études. Une évaluation plus complète de la force requise pour ouvrir la bouche est nécessaire afin de clarifier cette question.

Si la survenue du spasme du masséter était due à une augmentation excessive de la tension de repos, il serait raisonnable de s'attendre à ce que les patients présentant un risque accru de spasme du masséter puisse démontrer une augmentation de la tension de repos après administration de succinylcholine comparativement à d'autres patients. Saddler $e t$ al. ${ }^{3}$ ont étudié huit enfants atteints de strabisme, une condition associée à un risque accru de spasme du masséter. Ils n'ont pas trouvé de différence dans l'augmentation de la tension de repos après succinylcholine comparativement à d'autres enfants. Il s'ensuit que : soit ces enfants ne sont pas plus susceptibles de démontrer un spasme du masséter, soit que les facteurs prédisposants au spasme du masséter ne sont pas réflétés par ces mesures de la tension de repos. Dans une étude rétrospective récente déclarant une incidence accrue de spasme du masséter chez les enfants atteints de strabisme," le nombre de cas identifiés était petit et l'inci- dence de spasme du masséter était de 2.8 pour cent seulement soit 1 sur 35 . Si la puissance de ces mesures des variations de la tension de repos ${ }^{2,3}$ est similaire à celle de l'identification rétrospective du spasme du masséter ${ }^{11}$ il faudrait pour identifier un événement relié au spasme du masséter dans une population de huit, une incidence supérieure à 20 pour cent. Ces investigateurs n'étaient pas assez chanceux pour observer un événement rare dans une petite population de huit enfants.

Peut-être que le spasme du masséter n'est pas uniquement quantitativement différent des variations de la tension de repos mesurée ici, ${ }^{8,9}$ mais aussi qualitativement différent. S'il en est de même, les critères identifiants du spasme du masséter demeurent à être décrits avec suffisamment de détails pour permettre la différentiation du spasme du masséter avec une augmentation normale de la tension de repos après succinylcholine.

En résumé, ces trois études ${ }^{1-3}$ quantifient les aspects de la fonction neuromusculaire du masséter qui sont pertinents à l'usage efficace des bloqueurs neuromusculaires en anesthésie. Le dilemme de l'incidence relativement élevé du spasme du masséter ${ }^{11,12}$ l'incidence de 50 pour cent des biopsies musculaires démontrant une susceptibilité à l'hyperthermie maligne, chez les patients ayant une histoire de spasme du masséter, ${ }^{13-15}$ et l'incidence basse de l'hyperthermie maligne ${ }^{16}$ ne peut être résolue par ces seules études. Néanmoins, ces trois études ont contribué énormément à notre compréhension des effets des bloqueurs neuromusculaires chez les enfants par l'identification d'une augmentation dépendant de la dose dans la tension de repos du masséter et par la découverte des sensibilités relatives de l'adducteur du pouce et du masséter à la succinylcholine et aux bloqueurs neuromusculaires non-dépolarisants.

\section{References}

1 Saddler JM, Bevan JC, Plumley MH, Donati F, Bevan $D R$. The potency of atracurium on masseter and adductor pollicis muscles in children. Can J Anaesth 1989; 37: 26-30.

2 Plumley MH, Bevan JC, Saddler JM, Donati $F$, Bevan $D R$. Dose-related effects of succinylcholine on the adductor pollicis and masseter muscles in children. Can $\mathbf{J}$ Anaesth 1989; 37: 15-20.

3 Saddler JM, Bevan JC, Plumley MH, Polomeno RC, Donati $F$, Bevan $D R$. Jaw tension after succinylcholine in children undergoing strabismus surgery. Can J Anaesth 1989; 37: 21-25.

4 Caffrey RR, Warren $M L$, Becker $K E$. Neuromuscular blockade monitoring comparing the orbicularis oculi and adductor pollicis muscles. Anesthesiology 1986; 65: 95-7. 
5 Smith CE, Donati F, Bevan DR. Differential effects of pancuronium on masseter and adductor pollicis muscles in humans. Anesthesiology 1989; 71: 57-61.

6 Whittaker $M$. Cholinesterase. I $n$ : Monographs in Human Genetics, Vol. 11, New York: Karger, 1986.

7 DeCook TH, Goudsouzian NG. Tachyphylaxis and phase II block development during infusion of succinylcholine in children. Anesth Analg 1980; 59: 639-43.

8 Van Der Spek AFL, Fang WB, Ashton-Miller JA, Stohler CS, Carlson DS, Schork MA. The effects of succinylcholine on mouth opening. Anesthesiology 1987; 67: 459-65.

9 Van Der Spek AFL, Fang WB, Ashton-Miller JA, Stohler CS, Carlson DS, Schork MA. Increased masticatory muscle stiffness during limb muscle flaccidity associated with succinylcholine administration. Anesthesiology 1988; 69: 11-6.

10 Van Der Spek AFL, Reynolds PI, Ashton-Miller JA, Stohler CS, Schork MA. Differing effect of agonist and antagonist muscle relaxants on cat jaw muscles. Anesth Analg 1989; 69: 76-80.

11 Carroll JB. Increased incidence of masseter spasm in children with strabismus anesthetized with halothane and succinylcholine. Anesthesiology 1987; 67: 559-61.

12 Schwartz L, Rockoff MA, Koka BV. Masseter spasm with succinylcholine. Anesthesiology 1984; 61: 772-5.

13 Ellis FR, Halsall PJ. Suxamethonium spasm. A differential diagnostic conundrum. Br J Anaesth 1984; 56: 381-3.

14 Rosenberg $H$, Fletcher JE. Masseter muscle rigidity and malignant hyperthermia susceptibility. Anesth Analg 1986; 65: 161-4.

15 Ording $H$, Rankley $E$, Fletcher $R$. Investigation of malignant hyperthermia in Denmark and Sweden. Br J Anaesth 1984; 56: 1183-90.

16 Ording $H$. Incidence of malignant hyperthermia in Denmark. Anesth Analg 1985; 64: 700-5. 